\title{
Strongly interacting bosons in multi-chromatic potentials supporting mobility edges: localization, quasi-condensation and expansion dynamics
}

\author{
Pedro Ribeiro, Masudul Haque, and Achilleas Lazarides \\ Max-Planck-Institut für Physik komplexer Systeme, \\ Nöthnitzer Straße 38, D-01187 Dresden, Germany
}

\begin{abstract}
We provide an account of the static and dynamic properties of hard-core bosons in a onedimensional lattice subject to a multi-chromatic quasiperiodic potential for which the single-particle spectrum has mobility edges. We use the mapping from strongly interacting bosons to weakly interacting fermions, and provide exact numerical results for hard-core bosons in and out of equilibrium. In equilibrium, we find that the system behaves like a quasi-condensate (insulator) depending on whether the Fermi surface of the corresponding fermionic system lies in a spectral region where the single-particle states are delocalized (localized). We also study non-equilibrium expansion dynamics of initially trapped bosons, and demonstrate that the extent of partial localization is determined by the single-particle spectrum.
\end{abstract}

\section{INTRODUCTION}

The localization of quantum particles and waves in disordered media has been a key theme common to several fields of physics since Anderson's prediction of the effect half a century ago [1]. Among the different setups leading to localization effects, quasiperiodic potentials with incommensurate periods [2] provide a particularly attractive realization, as their mathematical simplicity allows for various exact results. A one-dimensional (1D) system subject to such a quasiperiodic potential (AubryAndre potential [2]) has a localization transition at a finite value of the potential strength, in contrast to a random potential which in $1 \mathrm{D}$ causes localization at infinitesimal strength. Recently, pioneering experiments have explored the physics of bosons [3,6] and light [7, 8] in such potentials.

The single-particle spectrum acquires additional structure upon modification of the quasiperiodic superlattice potentials e.g., when there are two superlattice potentials with different wavelengths each incommensurate with the lattice, or by other modifications of the basic AubryAndre (AA) potential [9 21]. In these cases, different energy regions of the single-particle spectrum might become localized at different strengths of the potential, so that at some strengths of the potential there are both localized and delocalized eigenstates. Such a structure is known as a mobility edge [9]. Mobility edges are wellknown to exist in truly random potentials, but only in higher dimension.

The interplay between disorder and interactions is a prominent theme in the study of strongly correlated systems. With the realization of quasiperiodic potentials hosting bosonic atoms [3 6, a natural question is the behavior of interacting bosons in quasiperiodic potentials. Refs. 22 38 have studied the Bose-Hubbard model in the AA potential for nonzero interactions. Some of these works [32 35, 37] have used the infinite-interaction or hard-core limit of the Bose-Hubbard model, where multiple occupancies are disallowed. The hard-core boson (HCB) model can be mapped onto free fermions, allow- ing numerically exact calculations for relatively large system sizes even in the absence of translation symmetry. Ground state, finite-temperature, and non-equilibrium properties of hard-core bosons in the AA potential have been analyzed in some detail in these studies [32 35, 37].

In this article, we address ground-state and nonequilibrium properties of hard-core bosons in multichromatic quasiperiodic potentials (extended AubryAndre models) where the single-particle spectrum displays mobility edges. Our main result concerning equilibrium properties is a connection between the behavior of the many-body system (insulating or quasi-condensate) and the location of the Fermi energy of the corresponding free-fermion system (which is the chemical potential for both the corresponding free-fermion system and for the $\mathrm{HCB}$ ). The system acts like a quasi-condensate (or insulator) when the chemical potential is in an energy region where the single-particle states are extended (or localized), irrespective of the nature of single-particle states lying below the chemical potential. This phenomenon is particularly puzzling in the case where the chemical potential is in a localized region but there are filled extended states at lower energies. One would expect superfluid properties due to extended states being occupied. We demonstrate this remarkable property, that the system behavior is determined by the location of the chemical potential, through the study of various quantities (quasicondensate fraction scaling, off-diagonal order, entanglement entropy, etc).

We also study the dynamics after release of an initially trapped cloud of hard-core bosons in multi-chromatic potentials. Expansion of initially confined many-body systems in potentials displaying mobility edges (higherdimensional random potential) has been explored recently in two experiments [39, 40], providing motivation for theoretical non-equilibrium calculations such as ours.

We will start in Section II] with a description of the model and some single-particle properties of multichromatic potentials. Section III presents various ground state properties, highlighting the connection to the position of the chemical potential. Section IV discusses the 

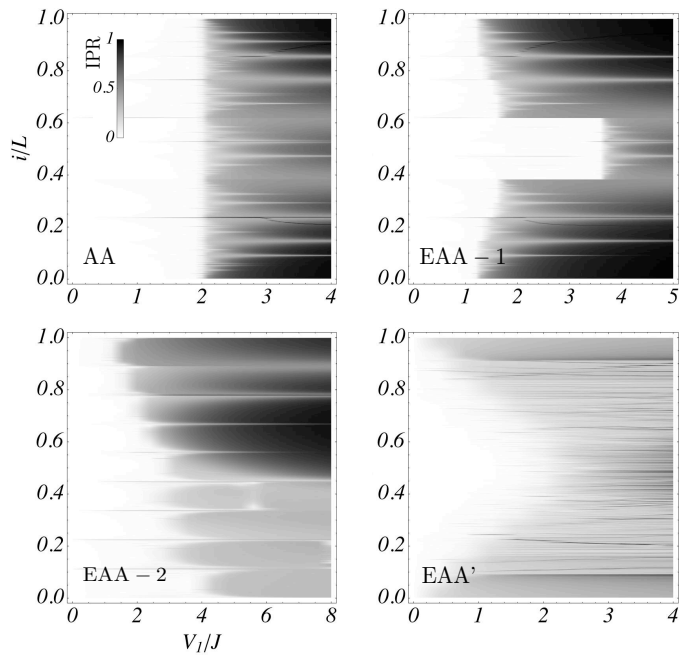

FIG. 1. Inverse participation ratio of all single-particle eigenstates, for the Aubry-Andre (AA) model and for the extended models EAA-1, EAA-2 and EAA'. Here $i$ is the eigenstate index in order of increasing eigenenergies. Except for AA, at intermediate potential strengths $V_{1}$ both localized and delocalized eigenstates coexist. Mobility edges are observed as a function of energy as one crosses boundaries between localized and extended regions.

non-equilibrium expansion dynamics.

\section{MODEL AND METHOD}

We consider a system of $N_{b}$ hard-core bosonic atoms on a chain with $L$ sites and open boundary conditions, described by the Hamiltonian

$$
H=-J \sum_{n}\left(b_{n}^{\dagger} b_{n+1}+b_{n+1}^{\dagger} b_{n}\right)+\sum_{n} V(n) b_{n}^{\dagger} b_{n}
$$

where $b_{n}$ and $b_{n}^{\dagger}$ are bosonic creation and annihilation operators, $\left[b_{n}, b_{n^{\prime}}^{\dagger}\right]=\delta_{n, n^{\prime}}$. The hard-core constraint is imposed through $b_{n}^{2}=b_{n}^{\dagger 2}=0$. We use several quasiperiodic potentials, $V(n)$, specified below.

Such a hard-core bosonic system may be regarded as the limit of the Bose-Hubbard model where the on-site Bose-Hubbard interaction is much larger than all other energy scales in the problem. This limit has the advantage of lending itself to exact calculations, through mapping to a fermionic system via the Jordan-Wigner (JW) transformation. Introducing the fermionic operators $c_{n}$ (with $\left\{c_{n}, c_{n^{\prime}}^{\dagger}\right\}=\delta_{n, n^{\prime}}$ ) the mapping

$$
b_{n}=S(0, n-1) c_{n},
$$

where $S\left(n, n^{\prime}\right)=\prod_{j=n}^{n^{\prime}}\left(1-2 n_{j}\right)$ is the JW string operator, reproduces the HCB commutation relations. After the JW mapping the fermionic Hamiltonian can be obtained from Eq. (1) replacing $b_{n} \rightarrow c_{n}$. The bosonic

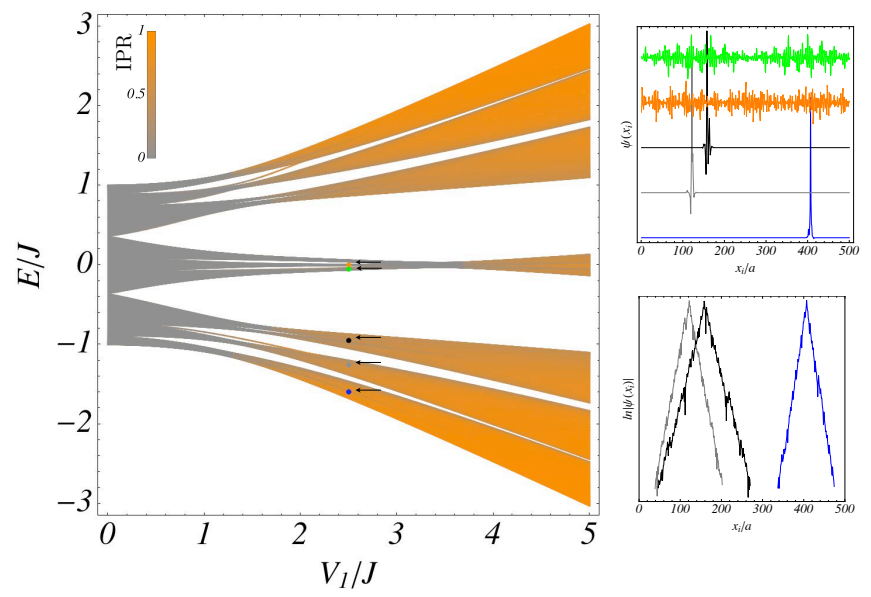

FIG. 2. Left: Single-particle spectrum as a function of the quasi-periodic potential amplitude for the EAA-1 model. The color code indicates IPR values. The localizationdelocalization transition occurs where many eigenstates bunch together; this bunching occurs at different $V_{1}$ for different parts of the spectrum. Upper right: some representative wave functions for $V_{1}=2.5 \mathrm{~J}$ (mobility edge region) for the states indicated with colored dots in left panel. Both localized and extended eigenfunctions are seen. Lower right: absolute value of the localized wave functions is plotted in log-linear scale showing that localization is exponential.

one-body density matrix

$$
\rho_{n n^{\prime}}^{B}=\left\langle b_{n}^{\dagger} b_{n^{\prime}}\right\rangle
$$

can be computed from the fermionic one, $\rho_{n n^{\prime}}^{F}=\left\langle c_{n}^{\dagger} c_{n^{\prime}}\right\rangle$, using the approach described, e.g., in Ref. 41.

For uniform 1D bosons at zero temperature, the occupancy of the lowest single-particle state (with momentum $k=0$ ) scales as $n_{k=0} \propto \sqrt{N_{b}}$. While Bose Einstein condensation (BEC) is not strictly present in $1 \mathrm{D}$, the off-diagonal elements of the density matrix still develop a algebraic decay $\rho_{n n^{\prime}}^{B} \propto \frac{1}{\left|n-n^{\prime}\right|^{1 / 2}}$ as $\left|n-n^{\prime}\right| \rightarrow \infty$ in the thermodynamic limit. In spatially inhomogeneous situations, the quantity analogous to $n_{k=0}$ is the largest eigenvalue $\lambda_{0}$ of the single-particle density matrix. The eigenvalues are referred to as occupation numbers of natural orbitals [41,43]. The natural orbitals are the corresponding eigenvectors:

$$
\sum_{j} \rho_{i j}^{B} \Phi_{j}^{n}=\lambda_{n} \Phi_{j}^{n}
$$

with $\lambda_{0} \geq \lambda_{1} \geq \ldots$. Quasi-condensation is signaled by the behavior $\lambda_{0} \propto \sqrt{N_{b}}$. Since we are dealing with explicitly non-uniform systems, we choose to use this language (rather than momentum occupancies) in order to describe the presence or absence of quasi-condensation.

We consider different types of quasi-periodic potentials. The simplest is a single-frequency cosine periodic potential with an irrational wavevector relative to the 
lattice spacing, generally known as the Aubry-Andre potential [2]:

$$
V(n)=V_{1} \cos \left(2 \pi q_{1} x_{n}\right) ; \quad \text { with } \quad q_{1}=\frac{\sqrt{5}-1}{2} a^{-1} .
$$

Here $x_{n}=$ an with $a$ the lattice constant. The singleparticle eigenstates of the AA model are all localized for $V_{1}>2$ and all extended for $V_{1}<2$. In this paper we are interested in extended Aubry-Andre models where the single-particle spectra have more intricate structure, in particular mobility edges. We introduce two potentials with two frequencies:

$$
V(n)=V_{1} \cos \left(2 \pi q_{1} x_{n}\right)+V_{2} \cos \left(2 \pi q_{2} x_{n}\right) .
$$

The first model has the parameters

$$
V_{2}=\frac{1}{6} V_{1}, q_{1}=\frac{\sqrt{5}-1}{2} a^{-1}, q_{2}=3 q_{1}
$$

and the other has parameters

$$
V_{2}=\frac{1}{3} V_{1}, q_{1}=\frac{0.7}{2 \pi} a^{-1}, q_{2}=2 q_{1} .
$$

We also consider another type of modification of the AA potential:

$$
\begin{array}{r}
V(n)=V_{1} \cos \left(2 \pi q_{1} x_{n}^{\alpha_{d}}\right) \\
q_{1}=\frac{\sqrt{5}-1}{2} a^{-1}, \alpha_{d}=0.7 .
\end{array}
$$

These models EAA-1,2 [9, 15] and EAA' [12] are known examples from a large class of extended Aubry-Andre models whose single-particle spectrum possess mobility edges.

As a function of the ratio $V_{1} / J$ these models present the same qualitative features: for small values of $V_{1} / J$ all the single particle eigenvectors are extended in real space and for large values of this ratio all the eigenstates are localized. For an intermediate range of $V_{1} / J$ both localized and delocalized eigenstates coexist. The single particle spectrum is organized in rather well defined regions corresponding to localized or extended eigenvectors separated by sharply defined mobility-edges. The generic behavior of the localized-extended transition is illustrated in Fig. 1 where the inverse participation ratio $\operatorname{IPR}(\psi)=\frac{\sum_{l}\left|\psi_{l}\right|^{4}}{\left[\sum_{l}\left|\psi_{l}\right|^{2}\right]^{2}}$ is plotted for the single-particle eigenstates of of $H$. Note that for the AA model there are no mobility edges in the spectrum, instead, the localizedextended transition occurs for all eigenstates simultaneously for the same value of $V_{1} / J$. This non-generic feature is due to the self-duality of the model [2]. Fig. 2 shows the single-particle spectrum as a function of $V_{1} / J$ and illustrates the difference between (exponentially) localized and extended states.

Fig. 1 illustrates that the models EAA-1, EAA-2 and EAA' present the same generic behavior. We therefore

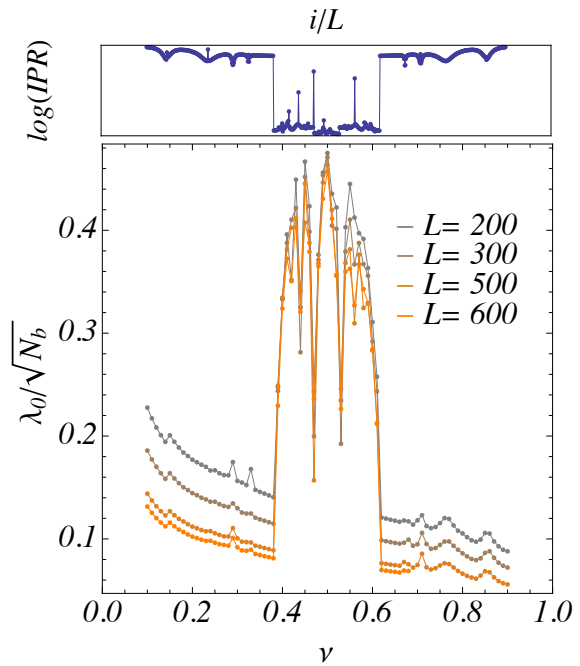

FIG. 3. Lower panel: quasi-condensate fraction given by $\lambda_{0} / \sqrt{N_{b}}$ as a function of the filling fraction $\nu=N_{b} / L$. $\left(V_{1}=2.5 \mathrm{~J}\right.$, EAA-1.) Quasi-condensation is observed when the chemical potential $\mu(\nu)$ lies in a spectral region with extended states (in this case for $\nu$ around half-filling, as seen from the top panel). Otherwise $\lambda_{0} / \sqrt{N_{b}}$ vanishes in the thermodynamic limit $N_{b} \rightarrow \infty$. Upper panel: inverse participation ratio against eigenstate index.

present our results mainly for one of the models (EAA-1). The expectation is that the concepts and results emerging from this work are generically valid for any potential for which the single-particle spectrum has mobility edges of this generic type.

\section{GROUND-STATE PROPERTIES}

In this Section we will present results characterizing the ground state properties of our system, as a function of the filling fraction $\nu=N_{b} / L$. The energy of the last filled JW fermionic level is the chemical potential of the system, $\mu(\nu)$. Our major result is that the system behaves like an insulator or a quasi-condensate, depending on whether $\mu(\nu)$ lies in an energy region of localized or extended single-particle states, which we will denote respectively by $\Sigma_{l}$ and $\Sigma_{e}$.

In order to characterize the ground state we consider the behavior of the natural orbitals occupation, the characteristic decay of the off-diagonal density matrix components, and the entanglement entropy of a subsystem. For definiteness we display results for the EEA-1 model with $V_{1}=5 / 2 J$, where the single-particle spectrum has a well-defined intermediate delocalized region $\left(\Sigma_{e}\right)$ separated by mobility edges from higher and lower energy regions of localized states $\left(\Sigma_{l}\right)$.

When all the single-particle eigenstates are localized (1D random potential or AA model with $V_{1}>2$ ), $\lambda_{0} / \sqrt{N_{b}} \rightarrow 0$ 33, 37], in contrast to the quasi-condensate behavior $\lambda_{0} \sim \sqrt{N_{b}}$. In addition, in the localized (insu- 


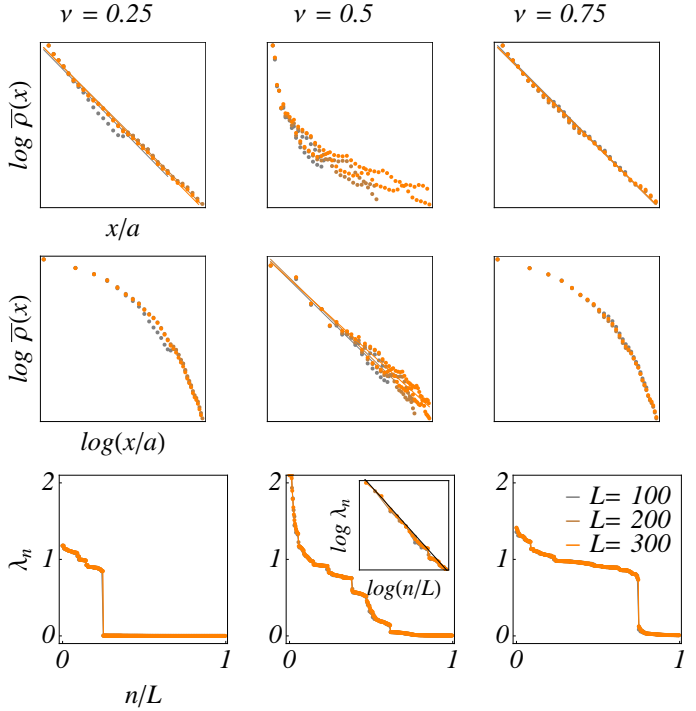

FIG. 4. Decay of the off-diagonal elements of $\rho^{B}$ for three different filling fractions in log-linear (upper row) and log-log (middle row) scales. $\left(V_{1}=2.5 \mathrm{~J}, \mathrm{EAA}-1\right.$.) Data points are shown for three different system sizes $L$ to indicate the degree of convergence. The straight lines are fits. For $\nu=0.25$ and $0.75\left[\mu(\nu) \in \Sigma_{l}\right.$, no quasi-condensation], the decay is seen to be exponential; $\bar{\rho}^{B}(x) \propto A e^{-|x| / \xi}$. For $\nu=0.5$ the decay is well approximated by power law $\bar{\rho}^{B}(x) \propto a|x|^{-\alpha}$ with $\alpha \simeq 0.59$. Lowest row: the natural orbital occupations $\lambda_{n}$. For $\nu=0.25$ and 0.75 the distribution is step-like. For $\nu=0.5$ it diverges for $n \rightarrow 0$ in the thermodynamic limit. This can be seen in the inset where the black line corresponds to a divergence of the form $\lambda_{n} \propto c(n / L)^{-\kappa}$ with $\kappa \simeq 0.3$.

lating) case, the off-diagonal correlations decay exponentially $\rho_{n n^{\prime}}^{B} \propto e^{-\left|n-n^{\prime}\right| / \xi}[33,37$, in contrast to the quasicondensate behavior $\rho_{n n^{\prime}}^{B} \propto \frac{1}{\left|n-n^{\prime}\right|^{1 / 2}}$. In the AA model the two behaviors are seen for $V_{1}>2$ an $V_{1}<2$ at any filling fraction 33 . We will show that when the singleparticle spectrum has mobility edges, either behavior can appear, depending on the filling.

Fig. 3 shows the rescaled lowest natural orbital occupation $\lambda_{0} / \sqrt{N_{b}}$ as a function of the filling fraction. The finite size scaling shows that if $\mu(\nu) \in \Sigma_{l}, \lambda_{0} / \sqrt{N_{b}} \rightarrow 0$ in the thermodynamic limit, while $\lambda_{0} / \sqrt{N_{b}}$ approaches a finite value for $\mu(\nu) \in \Sigma_{e}$.

The stark difference between the $\mu(\nu) \in \Sigma_{l}$ and $\mu(\nu) \in \Sigma_{e}$ cases is also observed in the off-diagonal elements of the averaged one-body density matrix $\bar{\rho}^{B}\left(x_{j}\right)=$ $\frac{1}{L} \sum_{i}^{L}\left|\rho_{i, i+j}^{B}\right|$ (see Figs. 4, 5. Here the average is taken to smoothen oscillations introduced by the quasi-periodic potential. For $\mu(\nu) \in \Sigma_{l}$ the decay is exponential $\bar{\rho}^{B}(x) \propto e^{-|x| / \xi}$ whether for $\mu(\nu) \in \Sigma_{e}$ one observes an algebraic behavior $\bar{\rho}^{B}(x) \propto|x|^{-\alpha}$. We found the exponent $\alpha$ to be in the range $\alpha \in[0.5,0.7]$ for our system sizes of $\mathcal{O}\left(10^{3}\right)$, for EAA-1, EAA-2, EAA' models. This is higher than in the bare tight-binding lattice $\left(V_{1}=0\right)$ case, for which $\alpha=1 / 2$ [1]. It is currently not (a)

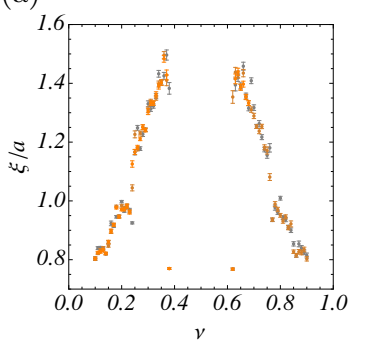

(b)

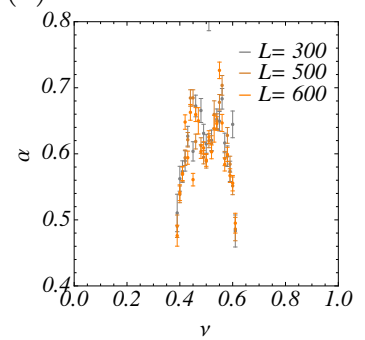

FIG. 5. $\quad\left(V_{1}=2.5 J\right.$, EAA-1. $)$ (a) Lengthscale $\xi$ of exponential decay of the off-diagonal elements of $\rho^{B}$, obtained by fitting with an exponential form $\bar{\rho}^{B}(x) \propto e^{-|x| / \xi}$ (see text). The error bars are given by the fit to the numerical data. For the $\nu$ values where quasi-condensation occurs, the behavior is algebraic (Fig. 4) and no $\xi$ values are shown. (b) Power-law exponent for off-diagonal decay $\bar{\rho}^{B}(x) \propto|x|^{-\alpha}$ in the region where quasi-condensation is present.

known whether $\alpha$ would converge to $1 / 2$ in the thermodynamic limit in multi-chromatic potentials with fillings corresponding to $\mu(\nu) \in \Sigma_{e}$.

The occupation of the natural orbitals, displayed in Fig 4 (lower panel), shows that for $\mu(\nu) \in \Sigma_{l}$ the distribution of the $\lambda_{n}$ 's is step-like, contrasting with the smooth decay for the $\mu(\nu) \in \Sigma_{e}$ case. Step-like features are usually assigned to fermionic distributions. Here the natural orbitals are localized 37] and the bosons behave much like in a fermionic system as no localized orbital can accommodate more than one $\mathrm{HCB}$.

Another striking difference between $\mu(\nu) \in \Sigma_{l}$ and $\mu(\nu) \in \Sigma_{e}$ cases is observed in the behavior of $\lambda_{n}$ at $n \rightarrow 0$. For $\mu(\nu) \in \Sigma_{e}$ one observes a divergence $\lambda_{n} \propto(n / L)^{-\kappa}$. For the example displayed in Fig $4, \kappa \simeq 0.3$. However this exponent does depends on $\nu$ and on the specific model of disorder and can even be larger the value $\kappa=1 / 2$ obtained for $V_{1,2}=0$. For $\mu(\nu) \in \Sigma_{l}$ no divergence is observed.

Finally, we consider scaling of the GS entanglement entropy of a subsystem $D$ as a function of the subsystem size $L_{D}$. The entanglement entropy is defined as $S_{D}=-\operatorname{Tr}_{D}\left[\hat{\rho}_{D} \ln \hat{\rho}_{D}\right]$, where $\hat{\rho}_{D}=\operatorname{Tr}_{\bar{D}}[\hat{\rho}]$ and $\hat{\rho}=\left|\Psi_{0}\right\rangle\left\langle\Psi_{0}\right|$ are many-body density matrices and $\operatorname{Tr}_{D}$ $\left(\operatorname{Tr}_{\bar{D}}\right)$ denote the trace over the degrees of freedom in $D$ (in the complement of $D$ ). For HCB the expression for the entanglement entropy simplifies 44 to $S_{D}=$ $-\sum_{i} \nu_{i} \ln \nu_{i}+\left(1-\nu_{i}\right) \ln \left(1-\nu_{i}\right)$ where the $\nu_{i}$ 's are the eigenvalues of the two-body density matrix of the JW fermions restricted to the subsystem $D, \rho_{i j}^{F}$ with $i, j \in D$. For $\mu(\nu) \in \Sigma_{l}, S_{D}$ saturates as $L_{D} \rightarrow \infty$. However for $\mu(\nu) \in \Sigma_{e}$ it behaves as $S_{D} \simeq \frac{\tilde{c}}{3} \ln L_{D}$ for large subsystem sizes $L_{D}$ with a prefactor $\tilde{c} \simeq 1$. This represents the well-known logarithmic correction to the "area law" in gapless one-dimensional systems [44, 45, with the prefactor given by the central charge $c=1$ in this case. Once again, the bosonic system behaves like a gapless quasicondensate or like a gapped insulator depending on the 


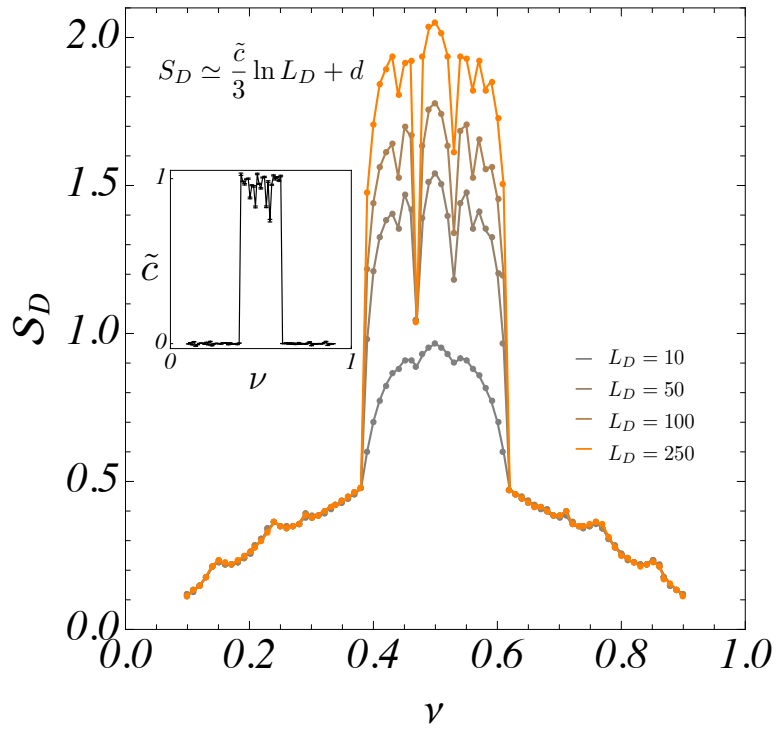

FIG. 6. Ground state entanglement entropy $S_{D}$ of a subsystem $D$, averaged over the central position of the subsystem, for various subsystem sizes $L_{D} .(L=1000$. $)$ For the $\nu$ values where quasi-condensation does not occur $\left[\mu(\nu) \in \Sigma_{l}\right]$, $S_{D}$ saturates to a constant value with increasing $L_{D}$. In the $\mu(\nu) \in \Sigma_{e}$ region, $S_{D}$ grows logarithmically with $L_{D}$. A $\log -$ arithmic fit (inset) shows that in this region the coefficient $\tilde{c}$ approaches the value $c=1$ which is the exact result for the case where no quasi-periodic potential is present.

location of the "Fermi energy" of the corresponding freefermion system.

\section{EXPANSION DYNAMICS}

In this section we address the non-equilibrium expansion dynamics of an initially trapped cloud of bosons after the trapping potential is turned off at time $t=0$. The motivation for studying this type of dynamical protocol is that expansion dynamics is particularly sensitive to the localization properties of the underlying single-particle states: particles will mostly fly off or mostly remain in the initial region depending on whether the initial overlap is dominantly with extended or localized single-particle states. This type of physics has also been explored in two recent experiments, which use higher-dimensional disordered potentials having mobility edges [39, 40.

The initial state $(t<0)$ is the ground state of the Hamiltonian (1) with an added harmonic trapping potential, i.e.,

$$
H(t)=H+\Theta(-t) \sum_{n} W(n) b_{n}^{\dagger} b_{n}
$$

where $\Theta(\ldots)$ is the Heaviside step function and

$$
W(n)=W\left(x_{n}-x_{n_{0}}\right)^{2},
$$

$n_{0}$ being a site near the center of the chain. We monitor the cloud size $\sigma(t)=\left[\left\langle\hat{x}_{i}^{2}(t)\right\rangle-\left\langle\hat{x}_{i}(t)\right\rangle^{2}\right]^{1 / 2}$ and the fraction of localized atoms $N_{\text {loc }}(t) / N_{b}$ as a function of time. Here, $N_{\text {loc }}(t)$ is defined as the number of atoms remaining in the support $S$ of the initial density distribution, $S=\left\{i:\left\langle\hat{n}_{i}(t=0)\right\rangle \neq 0\right\}$.

In the presence of a trapping potential and for $V_{1,2}=0$, a single dimensionless parameter $\tilde{\rho}=N_{b} \sqrt{W / J}$ controls the behavior of the atomic density in the large- $N_{b}$ limit [41. This means that $\rho_{n n}^{B} \rightarrow g\left(n \tilde{\rho} / N_{b} ; \tilde{\rho}\right) / N_{b}$ as $N_{b} \rightarrow$ $\infty$, where $g(y ; \tilde{\rho})$ is the normalized density distribution. For $\tilde{\rho} \simeq 2.6-2.7$ a Mott insulator region builds up in the middle of the trap, where the density is pinned to unity.

As in the last section we focus on the model EAA1. We study the dynamics for different values of $V_{1}$ and $\tilde{\rho}$. We separate the case $\tilde{\rho} \rightarrow \infty$, for which a simple interpretation of the results can be given, from the finite $\tilde{\rho}$ that leads to more complex dynamics.

\section{A. Large $\tilde{\rho}$}

We start by addressing the large $\tilde{\rho}$ limit where, in the initial density distribution, all the atoms are in the Mott phase. In other words, in the initial state a central block of the chain is completely filled while the rest of the chain is empty. Fig.7(a) shows snapshots of the time evolution of the initial density profile (left panel) for different values of $V_{1}$.

Figs. $7(\mathrm{~b}, \mathrm{c})$ display the time evolution of the cloud size and the fraction of localized particles. For $V_{1} \lesssim 3.8 \mathrm{~J}$, $\sigma(t) \propto v t$ (for $t \rightarrow \infty$ ), which means that at least some part of the cloud spreads ballistically. The spreading velocity $v$ is a decreasing function of $V_{1}$. The threshold value $V_{1} \simeq 3.8 t$ is that above which there are no more extended eigenstates in the spectrum, as can be seen from Fig.1(b). For $V_{1} \gtrsim 3.8 J, \sigma(t)$ saturates and the width of the cloud remains bounded for large times.

From Fig. 7)(c) one can see that, at long times, the fraction of particles that remain within the region $S$ is given by the fraction of extended states in the spectrum (horizontal dotted lines). This may be understood by considering the evolution in the eigenbasis of the nontrapped Hamiltonian $\left(W_{n}=0\right)$ with which the system evolves for $t>0$. In the asymptotic long time limit the fraction of delocalized atoms is proportional to the overlap of the initial state with the extended eigenstates of the non-trapped Hamiltonian. If the number of initially occupied sites is not too small, the initial state has approximately an equal overlap with all localized and extended eigenstates. The fraction of particles localized in $S$ should thus equal the fraction of extended eigenstates in the spectrum. 
(a)

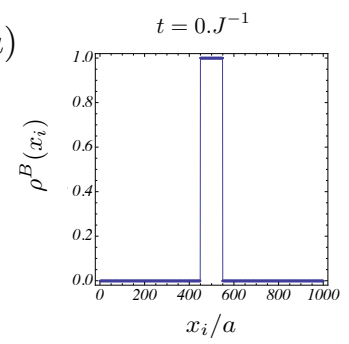

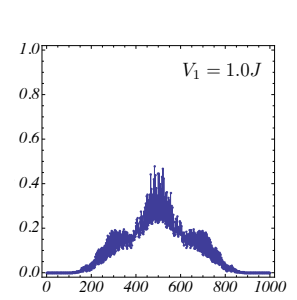

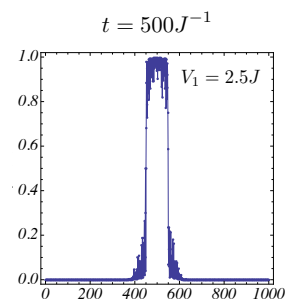

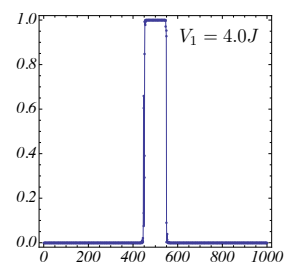
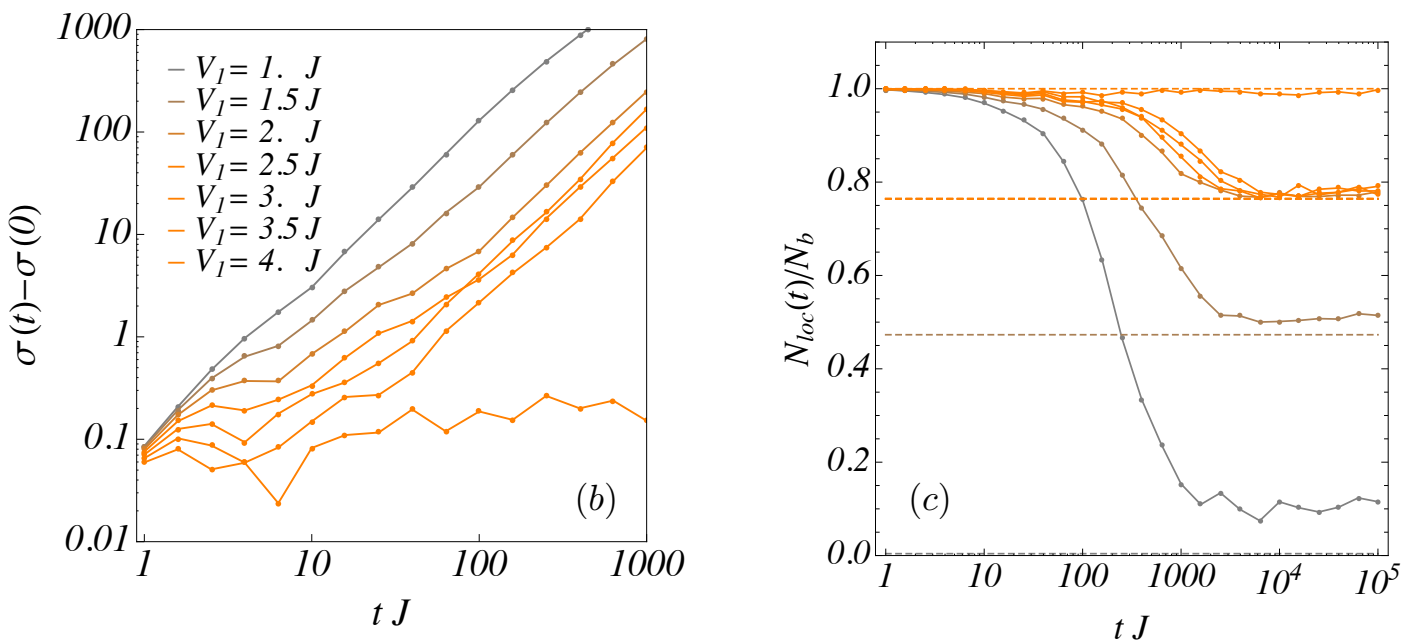

FIG. 7. Expansion dynamics of bosons initially clustered in the center of the lattice as shown in top left. $\left(N_{b}=100, L=1000\right.$. $)$ In the other (a) panels, the density profile at $t=500 J^{-1}$ is shown for $V_{1}=1 J, 2.5 J, 4 J$ (extended, mobility-edge, localized regimes). (b) Root-mean-square width of the expanding cloud. When there is a fraction of extended states and since they have a non-zero overlap with the initial state, the evolution is always ballistic for large times $\sigma(t) \propto v t$, nevertheless the pre-factor $v$ gets smaller with increasing $V_{1}$ as the fraction of localized eigenstates increases. When all the single particle states are localized the cloud width saturates for large times. (c) Time evolution of the number of bosons in the central region $S$ (see text). The dashed lines give the fraction of delocalized eigenstates in the single-particle spectrum.

\section{B. Arbitrary $\tilde{\rho}$}

Next, we address the time evolution starting from the ground state of a harmonic trapping potential characterized by a finite value of $\tilde{\rho}$. Fig. 8 shows the asymptotic long-time value of the fraction of atoms localized in $S$ as a function of $\tilde{\rho}$ for different values of $V_{1}$ and for $N_{b}=25,50,100$. The convergence of our results for increasing $N_{b}$ shows that the physics of the large- $N_{b}$ limit is well-represented in our numerical simulations and also that, for these $N_{b}$ values, effects of the location of the quasiperiodic potential is sufficiently averaged over.

For $\tilde{\rho}=0$ (no trap) and $V_{1} \gtrsim 1.2 \mathrm{~J}$ all the atoms are localized as the ground state is obtained by filling the first $N_{b}$ single-particle levels, which are all localized for the EAA-1 potential in this filling region. For small $\tilde{\rho}$ (very shallow trap) one can still find $N_{b}$ localized single-particle eigenstates within the trapping length $\ell=N_{b} a / \tilde{\rho}$. This explains why all bosons remain localized for small but nonzero values of $\tilde{\rho}$.

However, as $\tilde{\rho}$ increases, the number of localized states available within a region of size $\ell$ starts to be smaller than $N_{b}$ and the overlap with delocalized states is then finite.
For large $\tilde{\rho}$ the results of the last section are recovered and the fraction of localized atoms equals the fraction of localized states in the spectrum.

\section{SUMMARY \& DISCUSSION}

We have presented a study of a strongly interacting many-boson system in extended Aubry-Andre models for which the single-particle spectrum displays mobility edges, i.e., in situations where both extended and localized states are present in regions $\left(\Sigma_{e}\right.$ and $\left.\Sigma_{l}\right)$ of the single-particle spectrum. The bosonic system was treated by mapping to free fermions, so that numerically exact calculations are possible for relatively large systems. We have shown through non-equilibrium calculations that expansion dynamics can be used as a probe of the sizes of $\Sigma_{e}$ and $\Sigma_{l}$ regions in the single-particle spectrum.

For ground-state properties, the most striking result is that the properties of the many-body system (insulating or quasi-condensate) is determined solely by the location of the chemical potential $\mu(\nu)$, which has the interpre- 

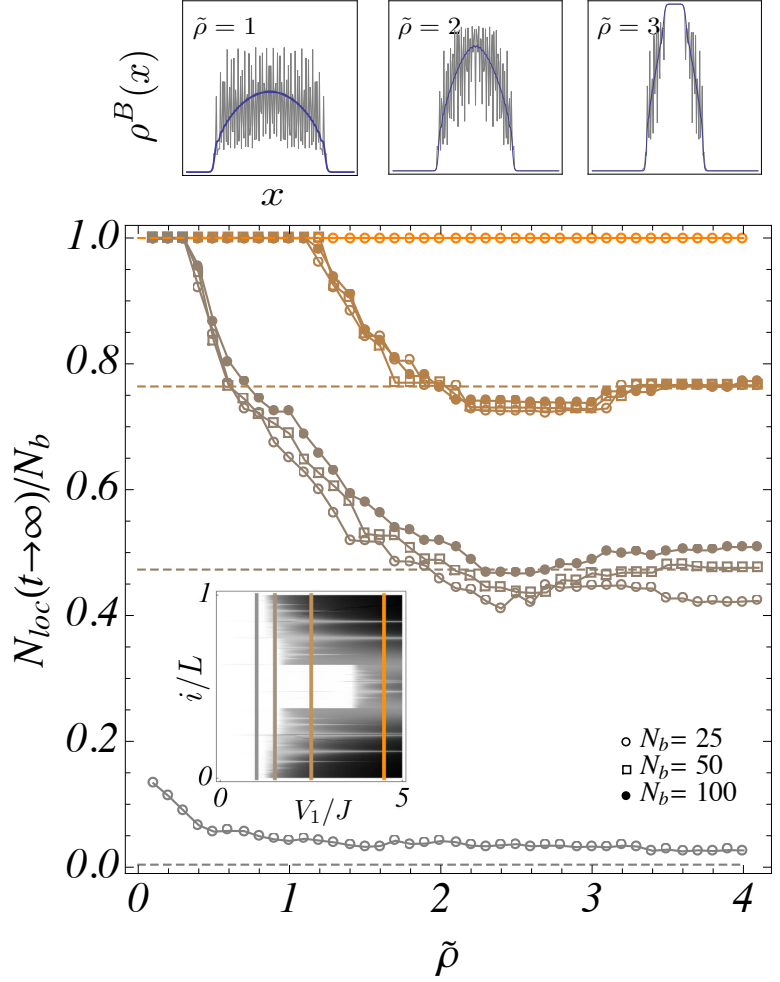

FIG. 8. Main panel: fraction of bosons remaining localized for asymptotically long times after switching off the trapping potential, as a function of parameter $\tilde{\rho}$. $(L=2000$, EAA1 , various $V_{1}$ and $N_{b}$.) For large $\tilde{\rho}$ the asymptotic value is given by the fraction of delocalized eigenstates in the spectrum (horizontal dashed lines). For small $\tilde{\rho}$ all the bosons remain localized after releasing the trap. The inset displays the IPR (as in Fig. 1); the colored lines correspond to the values of $V_{1} / J$ of the main panel. Upper panels: the initial density profile is displayed for $V_{1} / J=0$ (blue) and for $V_{1} / J=1$ (gray), for different values of $\tilde{\rho}$.

tation of being the Fermi energy of the corresponding free-fermion system. We have illustrated this by taking a representative model where, in a range of potential strengths, increasing the filling fraction $\nu$ takes the chemical potential from a $\mu(\nu) \in \Sigma_{l}$ region at low densities, through a region of $\mu(\nu) \in \Sigma_{e}$ region at intermediate $\nu$, to a $\mu(\nu) \in \Sigma_{l}$ region at high densities. For different fillings $\nu$, we presented the occupations of natural or- bitals $\left(\lambda_{n}\right.$, particularly the quasi-condensate occupancy $\left.\lambda_{0}\right)$, the off-diagonal decay of the single-particle bosonic density matrix, and the entanglement entropy scaling. These observables all show characteristics of gapped insulators in fillings for which $\mu(\nu) \in \Sigma_{l}$, and show characteristics of gapless quasi-condensates at $\nu$ values for which $\mu(\nu) \in \Sigma_{e}$.

At present we lack a simple explanation for this result that the location (relative to regions of the singleparticle spectrum) of the Fermi surface of the corresponding free-fermion system determines off-diagonal properties of strongly interacting bosons. While properties of fermionic systems are often described by features near the Fermi surface, the properties we have shown are bosonic rather than fermionic. Also, it is rather unintuitive that, when $\mu(\nu) \in \Sigma_{l}$, no quasi-condensate properties are seen even if a sizable fraction of Jordan-Wigner fermions occupy extended states.

Ref. 46] has observed, for another case (free fermions in disordered 2D Chern insulators) where the singleparticle states has different natures in different parts of the spectrum, that certain properties depend solely on the nature of the eigenstates at the location of the Fermi surface. This loosely similar observation in a very different context suggests that the situation is generic for systems where the single-particle spectrum contains regions of different nature.

These results open up several questions that deserve to be addressed in future research. Most prominently, one would like to know if this dependence on the location of the filling fraction extends far beyond the hard-core limit for bosons with strong but finite interactions (e.g., BoseHubbard chain with finite $U$ ). This would be particularly intriguing because such a system does not map exactly to free fermions, so that a simple picture of filling up the fermi sea does not hold. More speculatively, one might also wonder if bosons in higher dimensional lattices with mobility edges also have properties determined solely by the location of the chemical potential, since in higher dimensions there is no connection to a fermionic picture. Another interesting direction would be to study finitetemperature properties. Finite temperatures might allow the system to explore the $\Sigma_{l(e)}$ region even when the chemical potential is in the $\Sigma_{e(l)}$ region, if the chemical potential is sufficiently close to a mobility edge separating the two regions.
[1] P. W. Anderson, Phys. Rev. 109, 1492 (1958).

[2] S. Aubry and G. André, Ann. Israel Phys. Soc. 3, 133 (1980).

[3] G. Roati, C. D'Errico, L. Fallani, M. Fattori, C. Fort, M. Zaccanti, G. Modugno, M. Modugno, and M. Inguscio, Nature 453, 895 (2008).

[4] J. E. Lye, L. Fallani, C. Fort, V. Guarrera, D. S. Modugno, M. Wiersma, and M. Inguscio, Phys. Rev. A. 75,
061603 (2007).

[5] L. Fallani, J. E. Lye, V. Guarrera, C. Fort, K. M. R. van der Stam, and M. Inguscio, Phys. Rev. Lett. 98, 130404 (2007).

[6] V. Guarrera, N. Fabbri, L. Fallani, C. Fort, K. M. R. van der Stam, and M. Inguscio, Phys. Rev. Lett. 100, 250403 (2008).

[7] Y. Lahini, R. Pugatch, F. Pozzi, M. Sorel, R. Morandotti, 
N. Davidson, and Y. Silberberg, Phys. Rev. Lett. 103, 013901 (2009).

[8] Y. E. Kraus, Y. Lahini, Z. Ringer, M. Verbin, and O. Zilberberg, Phys. Rev. Lett. 109, 106402 (2012).

[9] C. M. Soukoulis and E. N. Economou, Phys. Rev. Lett. 48, 1043 (1982).

[10] R. Riklund, Y. Liu, G. Wahlstrom, and Z. Zhao-bo, Journal of Physics C: Solid State Physics 19, L705 (1986).

[11] S. Das Sarma, A. Kobayashi, and R. E. Prange, Phys. Rev. Lett. 56, 1280 (1986)

[12] S. Das Sarma, S. He, and X. C. Xie, Phys. Rev. Lett. 61, 2144 (1988).

[13] X. C. Xie and S. Das Sarma, Phys. Rev. Lett. 60, 1585 (1988).

114 M. Griniasty and S. Fishman, Phys. Rev. Lett. 60, 1334 (1988)

[15] H. Hiramoto and M. Kohmoto, Phys. Rev. B 40, 8225 (1989).

[16] I. Varga, J. Pipek, and B. Vasvári, Phys. Rev. B 46, 4978 (1992)

[17] V. W. Scarola and S. Das Sarma, Phys. Rev. A 73, 041609 (2006)

[18] D. J. Boers, B. Goedeke, D. Hinrichs, and M. Holthaus, Phys. Rev. A 75, 063404 (2007)

[19] J. Biddle, B. Wang, D. J. Priour, and S. Das Sarma, Phys. Rev. A 80, 021603 (2010).

[20] J. Biddle and S. Das Sarma, Phys. Rev. Lett. 104, 070601 (2010).

[21] J. Biddle, D. J. Priour, B. Wang, and S. Das Sarma, Phys. Rev. B 83, 075105 (2011).

[22] G. Roux, T. Barthel, I. P. McCulloch, C. Kollath, U. Schollwöck, and T. Giamarchi, Phys. Rev. A 78, 023628 (2008).

[23] X. Deng, R. Citro, A. Minguzzi, and E. Orignac, Phys. Rev. A 78, 013625 (2008).

[24] X. Deng, R. Citro, E. Orignac, and A. Minguzzi, The European Physical Journal B - Condensed Matter and Complex Systems 68, 435 (2009).

[25] G. Orso, A. Iucci, M. A. Cazalilla, and T. Giamarchi, Phys. Rev. A 80, 033625 (2009).
[26] F. Schmitt, M. Hild, and R. Roth, Phys. Rev. A 80, 023621 (2009)

[27] J. Zakrzewski and D. Delande, Phys. Rev. A 80, 013602 (2009).

[28] L. Sanchez-Palencia and M. Lewenstein, Nature Physics 6, 87 (2010).

[29] T. Roscilde, Phys. Rev. A 82, 023601 (2010).

[30] U. Shrestha and M. Modugno, Phys. Rev. A 82, 033604 (2010).

[31] K. He, I. I. Satija, C. W. Clark, A. M. Rey, and M. Rigol, Phys. Rev. A 85, 013617 (2012).

[32] X. Cai, S. Chen, and Y. Wang, Phys. Rev. A 83, 043613 (2011).

[33] X. Cai, S. Chen, and Y. Wang, Phys. Rev. A 81, 023626 (2010).

[34] X. Cai, S. Chen, and Y. Wang, Phys. Rev. A 81, 053629 (2010).

[35] L.-J. Lang, X. Cai, and S. Chen, Phys. Rev. Lett. 108, $220401(2012)$

[36] A. Cetoli and E. Lundh, EPL 90, 46001 (2010).

[37] N. Nessi and A. Iucci, Phys. Rev. A 84, 063614 (2011).

[38] S. Flach, M. Ivanchenko, and R. Khomeriki, EPL 98, 66002 (2012)

[39] S. S. Kondov, W. R. McGehee, J. J. Zirbel, and B. DeMarco, Science 334, 66 (2011)

[40] F. Jendrzejewski, A. Bernard, K. Müller, P. Cheinet, V. Josse, M. Piraud, L. Pezzé, L. Sanchez-Palencia, A. Aspect, and P. Bouyer, Nature Physics 8, 398-403 (2012).

[41] M. Rigol and A. Muramatsu, Phys. Rev. A 72, 013604 (2005).

[42] O. Penrose and L. Onsager, Phys. Rev. 104, 576 (1956).

[43] A. J. Leggett, Rev. Mod. Phys. 73, 307 (2001).

[44] G. Vidal, J. I. Latorre, E. Rico, and A. Kitaev, Phys. Rev. Lett. 90, 227902 (2003).

[45] P. Calabrese and J. Cardy, J. Stat. Mech. 2004, P06002 (2004).

[46] E. Prodan, T. L. Hughes, and B. A. Bernevig, Phys. Rev. Lett. 105, 115501 (2010). 